\title{
EHR Conformance Testing for Enhanced Syndromic Surveillance System Interoperability
}

\author{
Robert Snelick', Charlie Ishikawa², Rebecca Zwickl and Sheryl Taylor*3 \\ ${ }^{1}$ National Institute of Standards and Technology, Gaithersburg, MD, USA; ${ }^{2}$ International Society for Disease Surveillance, Brighton, \\ MA, USA; ${ }^{3}$ Booz Allen Hamilton, Vienna, VA, USA
}

\section{Objective}

Describe how the National Institute of Standards and Technology's (NIST) Syndromic Surveillance Messaging Validation Suite supports federal efforts to increase electronic health record (EHR) interoperability for timelier public health surveillance capabilities in the US.

\section{Introduction}

Lack of speed, reliability, and uniformity of data collection limit the ability of syndromic surveillance (SyS) systems to provide public health authorities (PHAs) with timely information on community health threats and trends. Electronic information technologies have long been used to accelerate and automate data collection for more real-time surveillance. There is, however, irregularity in how SyS data are packaged and sent by healthcare providers. In the US, federal programs to improve patient and population health outcomes are promoting enhanced EHR technology interoperability with a newly mandated standard for SyS data communication. Under a federal rule tied to Medicare and Medicaid reimbursement rates, hospitals are now required to provide SyS data to PHAs using HL7 2.5.1 messages that are in conformance with the CDC's Public Health Information Network (PHIN) guide for SyS. Merely mandating this standard, however, does not necessarily mean that it will be used as intended or that EHR SyS data will be interoperable among systems. Technology standards are frequently implemented with inconsistencies that spring from guidance ambiguities or misinterpretations. Inserting thorough conformance testing early in the technology development life cycle can increase the probability of conformance to standards, interoperability, and product reliability while reducing overall costs.

\section{Methods}

To improve the probability of conformance to standards and to promote interoperability, the Office of the National Coordinator for Health Information Technology (ONC) maintains a voluntary EHR certification program that validates SyS data messages from EHRs against the mandated standard. The certification testing employs a context-based validation method using a suite of publicly accessible, web-based tools developed by subject matter experts from NIST, ISDS, and the CDC. This SyS Messaging Validation Suite (SySMVS) includes SyS message validation services, test stories that set the clinical context, test SyS data, testing guidance, and a forum for testers to ask questions or provide feedback about the suite. ONC-Accredited Testing Laboratories (ATLs) use the tools to test EHR technologies for certification, and EHR vendors can use the tools while developing their technologies. Healthcare providers and PHAs can use the validation suite to verify conformance of SyS messages containing real patient data for implementing SyS data exchange.

\section{Results}

As of August, 2013, thirty-five vendors had certified their EHR technologies for SyS messaging. The ATLs tested these EHRs using the SyS-MVS. Feedback about these tools indicates that EHR technology vendors and other groups: (1) have been motivated to think through the steps for capturing/reporting syndromic data in more detail than before; (2) are challenged by the new SyS data requirements and standards; (3) appreciate the subject matter expertise that generated the tools; and (4) plan to use these tools locally to help real-world implementations.

\section{Conclusions}

The SyS-MVS, used nationally by the ATLs to test EHR technologies for certification and locally by healthcare providers to validate electronic messages before sending their SyS data to PHAs, is helping to drive the industry toward the level of interoperability needed to promote efficient reporting and utilization of syndrome-based public health surveillance information.

\section{Keywords}

certification testing; electronic health records; meaningful use; conformance testing; standards

\author{
*Sheryl Taylor \\ E-mail: taylor_sheryl@bah.com
}

